H. Ergun, J. Dave, D. Van Hertem and F. Geth, "Optimal Power Flow for AC/DC Grids: Formulation, Convex Relaxation, Linear Approximation and Implementation," in IEEE Transactions on Power Systems (Early Access).

Digital Object Identifier: 10.1109/TPWRS.2019.2897835

URL: $\underline{\text { http://ieeexplore.ieee.org/stamp/stamp.jsp?tp=\&arnumber }=8636236 \text { \&isnumber }=4374138}$

(C) 2019 IEEE. Personal use of this material is permitted. Permission from IEEE must be obtained for all other users, including reprinting/ republishing this material for advertising or promotional purposes, creating new collective works for resale or redistribution to servers or lists, or reuse of any copyrighted components of this work in other works. 


\title{
Optimal Power Flow for AC/DC Grids: Formulation, Convex Relaxation, Linear Approximation and Implementation
}

\author{
Hakan Ergun, Member, IEEE, Jay Dave, GSM, IEEE, Dirk Van Hertem, Senior Member, IEEE, \\ and Frederik Geth, Member, IEEE
}

\begin{abstract}
HVDC is becoming an increasingly important part of the present day transmission systems. Accurate models of active and reactive power control capabilities of HVDC converter stations are required to analyse the operation of power systems consisting of ac and dc grids, including ancillary services and security. Different converter station technologies exist, with varying control characteristics. This paper develops an optimal power flow model for ac and dc grids. A variety of formulations, from non-linear to convexified to linearized, are developed and implemented in an open-source tool. A convex relaxation formulation of a parameterized ac/dc converter model is developed. The hierarchy of common ac optimal power flow formulations is mapped to formulations for converter stations and dc grids. Numerical illustrations for a number of test cases, up to 3120 ac nodes and up to $10 \mathrm{dc}$ nodes and converters, are provided.
\end{abstract}

Index Terms-HVDC transmission, flexible ac transmission systems, power system analysis computing

\section{INTRODUCTION}

\section{A. Background}

In recent years, high voltage direct current (HVDC) grids gained on interest due to the use of voltage-source (VSC) and modular multilevel (MMC) converters allowing HVDC systems to be operated in multi-terminal and/or meshed grid configurations. Especially in Europe, where many offshore wind farms are connected via VSC HVDC to the main network, the feasibility of a European offshore HVDC grid is currently analyzed from several different perspectives [1]-[4]. Also in India and China, HVDC has become a focal part of the transmission system. HVDC systems can enhance system security and deliver ancillary services to the ac transmission system, by means of their active and reactive power control capabilities. VSC converters are able to use independent active and reactive power set points, which helps to achieve power flow and voltage control within ac grids. In order to assess the performance of an HVDC system while accurately modeling the steady state system behavior, a robust ac/dc optimal power flow (OPF) methodology is required.

H. Ergun, J. Dave, and D. Van Hertem are with the Research Group ELECTA, Department of Electrical Engineering, KU Leuven, Leuven 3000, Belgium (e-mail: hakan.ergun @kuleuven.be) and with EnergyVille, Genk, Belgium.

F. Geth is with CSIRO Energy, Newcastle NSW Australia (frederik.geth@csiro.au).

Special thanks to dr. Jef Beerten for his valuable input.

\section{B. Literature review}

Several publications discuss the power flow problem formulation in the context of ac/dc grids. In [5] a power flow algorithm for combined ac and dc grids is introduced using non-linear power flow equations. The power flow problem is solved iteratively, solving the ac and dc grid equations simultaneously in each step. Converter transformer and filters are taken into account but power-electronic losses are not. A comprehensive power flow algorithm for meshed ac and $\mathrm{dc}$ grids is introduced in [6], which solves the non-linear $\mathrm{dc}$ and ac grid equations sequentially. A detailed converter model including converter transformer, filters and quadratic converter losses is used.

In [7] a first optimal power flow problem algorithm is introduced for ac grids incorporating point-to-point HVDC connections, which are modeled as bus injections on the ac grid. The OPF is formulated as a quadratic programming problem but converter losses, converter transformers and filters are neglected. Ref. [8] introduces a non-linear ac/dc OPF including quadratic converter losses, where converter transformer and filters are excluded. In [9] a similar nonlinear ac and dc grid OPF formulation is used, including a quadratic loss model for HVDC converters. In [10] an ac/dc OPF algorithm is proposed, using the non-linear power flow equations, iteratively solving the equations for the ac and the dc grid, building on Matpower [11] with its interior-point solver. Converters are modeled with quadratic losses, whereas converter transformers and filters are neglected. Also in [12], a similar OPF formulation is used to determine the costs and benefits of meshed HVDC grids, incorporating quadratic VSC converter losses. In [13] linearized formulations of the ac and dc grids equations have been used. Ref. [14] uses linearized formulations for the ac and dc grid including penalty terms for the ac voltage angle difference as well as the dc voltage magnitude difference across ac and dc branches, respectively.

Solving the classic nonconvex, and complex-valued ac OPF in practice depends on the conversion of the equations into real-valued equivalents which then are passed to mathematical optimization solvers. Non-linear programming solvers can be used to optimize continuous versions of the above-mentioned ac/dc OPF problems. Nevertheless, due to nonconvexity, such an approach suffers from a lack of convergence guarantee and inability to certify global optimality. Therefore, in order to solve a variety of problems efficiently, a hierarchy of 
OPF formulations has been developed in the context of ac grids. Next to the nonconvex approach, formulations include different convex relaxations and linear approximations in different variable spaces [15]. It is noted that formulations in the literature can be split into two major categories: bus injection models (BIMs) and branch flow models (BFMs) , [16].

In [17] a second order cone (SOC) programming relaxation of dc grid power flow equations is developed providing conditions under which this relaxation is exact. Ref. [18] introduces an ac/dc OPF with discrete transformer taps of the HVDC converter transformer. The method uses a combination of linearization and SOC relaxation of the ac and dc grid power flow equations, and includes line commutated converter (LCC) stations with a fixed voltage modulation factor. The optimal discrete tap positions are obtained by sequentially solving an OPF with fixed tap settings and then solving a linearized mixed integer OPF for optimal tap settings with sequential ac feasibility checks. Refs. [19], [20] develop a BIM semidefinite relaxation of ac/dc OPF, which is compared to Matpower [11].

\section{Scope}

Many of the ac/dc solution approaches either rely on the sequential solution of the ac and dc grid equations, or make simplifications in the converter model to solve the combined non-linear ac/dc OPF problem. This paper develops a single and comprehensive ac/dc OPF model and tool, providing nonlinear formulations, convex relaxations and linear approximations of the ac and dc grid power flow equations, allowing to pose OPF problems based on one integrated model. To the authors' knowledge there is no integrated optimal power flow model and implementation for ac/dc grids which allows the assessment of the performance of different relaxation schemes. Consistent power flow representations between the ac and dc grid formulations allow to explore the trade-offs between accuracy and speed for problems of different sizes. As such, a generic HVDC converter station model including transformers, phase reactors and filters, is used to represent LCC, VSC and MMC type converters. The accompanying open source implementation can easily be extended further with different power flow formulations and problem types.

HVDC converters are modeled with a quadratic loss model, and the losses of the converter transformer, filter and reactor have been taken into account as well. The converter station model is fully parametrized. For instance, instead of having to use small impedance values to represent missingness of components, which often leads to numerical issues, components can actually be omitted.

The ac/dc OPF model is implemented as an extension of the 'PowerModels.jl' [21] package (v0.8) built on top of Julia/JuMP [22]. As such, the existing ac grid formulations of PowerModels are used to define the flows in the ac grid part(s). The package is extended using a set of dc grid nodes, and with ac/dc converter stations to connect the dc grid nodes to the ac grid nodes. The implementation is then validated against existing tools for edge cases. An implementation of the formulations is made open source in 'PowerModelsACDC.j1', available at [23].
TABLE I

DEFINITION OF AC/DC OPF ENTITIES, INDICES AND SETS

\begin{tabular}{ll}
\hline ac nodes & $i, j \in \mathcal{I}$ \\
ac branches & $l \in \mathcal{L}$ \\
ac topology & $l i j \in \mathcal{T}^{\mathrm{ac}} \subseteq \mathcal{L} \times \mathcal{I} \times \mathcal{I}$ \\
dc nodes & $e, f \in \mathcal{E}$ \\
dc branches & $d \in \mathcal{D}$ \\
dc topology & def $\in \mathcal{T}^{\mathrm{dc}} \subseteq \mathcal{D} \times \mathcal{E} \times \mathcal{E}$ \\
ac/dc converters & $c \in \mathcal{C}$ \\
ac/dc converter topology & cie $\in \mathcal{T}^{\mathrm{cv}} \subseteq \mathcal{C} \times \mathcal{I} \times \mathcal{E}$ \\
generators & $g \in \mathcal{G}$ \\
loads & $m \in \mathcal{M}$ \\
ac generator connectivity & $g i \in \mathcal{T}^{\text {gen,ac }} \subseteq \mathcal{G} \times \mathcal{I}$ \\
ac load connectivity & $m i \in \mathcal{T}^{\text {load,ac }} \subseteq \mathcal{M} \times \mathcal{I}$ \\
dc load connectivity & $m e \in \mathcal{T}^{\text {load,dc } \subseteq \mathcal{M} \times \mathcal{E}}$ \\
\hline
\end{tabular}

The paper is structured as follows. In section II, the reference non-linear model is derived. Sections IV and III provide the linear approximation and the convex relaxations, respectively. Section V provides formulation properties and their comparison. Section VI provides numerical experiments and in section VII conclusions are drawn.

\section{NON-LINEAR AC/DC OPF MODEL}

Table I introduces the sets and indices used in the ac/dc OPF problem definition, which are used throughout the article. Some variables and equations are defined over dc branches with reversed node order. This set is defined as:

$$
\mathcal{T}^{\mathrm{dc}, \mathrm{rev}}=\left\{d f e: \text { def } \in \mathcal{T}^{\mathrm{dc}}\right\} .
$$

It is assumed that both ac nodal voltage magnitudes, $U_{i}^{\text {mag }}$ and dc node voltages, $U_{e}^{\mathrm{dc}}$ are subject to operational limits:

$$
\begin{gathered}
U_{i}^{\text {mag,min }} \leq U_{i}^{\text {mag }} \leq U_{i}^{\text {mag,max }} \quad \forall i \in \mathcal{I}, \\
U_{e}^{\mathrm{dc}, \max } \leq U_{e}^{\mathrm{dc}} \leq U_{e}^{\mathrm{dc}, \max } \quad \forall e \in \mathcal{E} .
\end{gathered}
$$

\section{A. Static DC Grid Model}

The dc branches are modeled according to Fig. 1, where in the single line representation the power flow over the branch is divided by the number of poles $p_{d} \in\{1,2\}$. For monopolar HVDC links, the entire power flow will be seen on the single pole, whereas for symmetrical monopole and bipolar HVDC links, the power flow will be divided between the positive and the negative pole [24]. This affects the Ohm's losses in the dc branch. Note that, even though in VSC grids dc branches have a significant capacitance and in LCC grids branches have significant inductance, this does not translate to shunt susceptance or series reactance in the branch model, as these terms are absent under the static power flow assumption.

The generic power flow model for the dc branches is defined as

$$
P_{d e f}^{\mathrm{dc}}+P_{d f e}^{\mathrm{dc}}=P_{d}^{\mathrm{dc}, \text { loss }} \quad \forall d e f \in \mathcal{T}^{\mathrm{dc}} .
$$

In physical systems the resistance is nonnegative $r_{d}^{\mathrm{s}} \geq 0$, and therefore branch losses are nonnegative ${ }^{1}$, i.e. $P_{d}^{\text {dc,loss }} \geq 0$. The power flow is bound by the ratings of the dc branches at both ends:

$$
-P_{d}^{\mathrm{dc}, \text { rated }} \leq P_{d e f}^{\mathrm{dc}}, \leq P_{d}^{\mathrm{dc}, \text { rated }} \forall \text { def } \in \mathcal{T}^{\mathrm{dc}} \cup \mathcal{T}^{\mathrm{dc}, \text { rev }} .
$$

${ }^{1}$ the implementation of the mathematical model does not enforce $r_{d}^{\mathrm{s}} \geq 0$ though. 


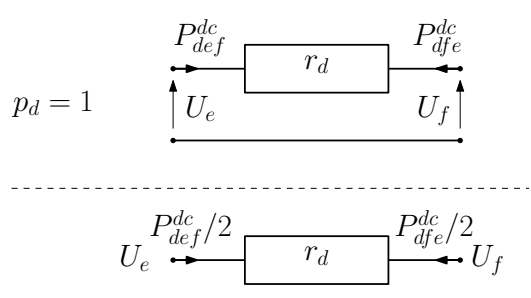

$p_{d}=2$

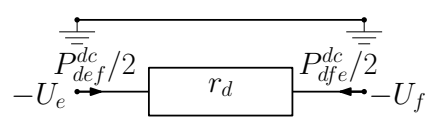

Fig. 1. Static de branch model.

1) Branch Flow Model: The power flow on the dc branches is defined as

$$
\begin{gathered}
P_{d e f}^{\mathrm{dc}}=p_{d} \cdot U_{e}^{\mathrm{dc}} \cdot I_{d e f}^{\mathrm{dc}} \quad \forall \operatorname{def} \in \mathcal{T}^{\mathrm{dc}}, \\
P_{d}^{\mathrm{dc}, \text { loss }}=p_{d} \cdot r_{d} \cdot\left(I_{d e f}^{\mathrm{dc}}\right)^{2} \quad \forall \text { def } \in \mathcal{T}^{\mathrm{dc}}, \\
-I_{d}^{\mathrm{dc}, \text { rated }} \leq I_{d e f}^{\mathrm{dc}} \leq I_{d}^{\mathrm{dc}, \text { rated }} \quad \forall \text { def } \in \mathcal{T}^{\mathrm{dc}} .
\end{gathered}
$$

where $P_{d e f}^{\mathrm{dc}}$ is the power flow on dc line $d$ going from dc node $e$ to $f$ and $P_{d f e}^{\mathrm{dc}}$ is the power flow in the opposite direction. The corresponding current variables are $I_{d e f}^{\mathrm{dc}}$ and $I_{d f e}^{\mathrm{dc}}$, with $I_{d e f}^{\mathrm{dc}}+I_{d f e}^{\mathrm{dc}}=0$. Furthermore, $U_{e}^{\mathrm{dc}}$ is the voltage magnitude of the dc node $e$.

2) Bus Injection Model: The BIM formulation of the dc line is

$$
P_{d e f}^{\mathrm{dc}}=p_{d} \cdot g_{d}^{\mathrm{s}} \cdot U_{e}^{\mathrm{dc}} \cdot\left(U_{e}^{\mathrm{dc}}-U_{f}^{\mathrm{dc}}\right) \forall \operatorname{def} \in \mathcal{T}^{\mathrm{dc}} \cup \mathcal{T}^{\mathrm{dc}, \mathrm{rev}},
$$

with $U_{f}^{\mathrm{dc}}$ the voltage of node $f$ and $g_{d}^{\mathrm{s}}=1 / r_{d}^{\mathrm{s}}$ series conductance of the dc branch $d$.

\section{B. Static AC/DC Converter Station Model}

The generic ac/dc converter station model is composed of (1) a transformer with tap and series impedance, (2) a filter as a shunt susceptance, (3) a phase reactor as series impedance and (4) a power-electronic ac/dc converter.

The transformer, filter and phase reactor are passive components, whereas the power-electronic converter is active. The passive components are described using the classic power flow model. The converter may be LCC, VSC or MMC, and may operate as an inverter and/or rectifier. Note that there is no Ohm's law defined over the converter, as it is an active component, i.e. the voltages at either side of the converter are only linked through conservation of energy (power balance). Note that the ac side has a complex-value voltage $U_{i}=U_{i}^{\mathrm{mag}} \angle \theta_{i}$ and the dc side has a real-value voltage (magnitude) $U_{e}^{\mathrm{dc}}$. The converter station also has two internal ac voltages, one at the filter, $U_{c}^{\mathrm{f}}=U_{c}^{\mathrm{f}, \mathrm{mag}} \angle \theta_{c}^{\mathrm{f}}$ and another one at the power-electronic converter $U_{c}^{\mathrm{cv}}=U_{c}^{\mathrm{cv}, \mathrm{mag}} \angle \theta_{c}^{\mathrm{cv}}$. An offnominal voltage transformation ratio can be used to enhance the controllability of the converter stations [25], [26]. Finally, w.r.t. the main ac grid, the passive components in the converter station are part of a radial branch. This furthermore means that any phase shift over the converter transformer would not influence the power flow, but merely apply an angle offset to
$U_{c}^{\mathrm{f}}$ and $U_{c}^{\mathrm{cv}}$ w.r.t. $U_{i}$. Therefore, such phase shift is neglected to simplify the derivation of the mathematical model. Fig. 2 summarizes the adopted converter station model and maps the variable symbols.

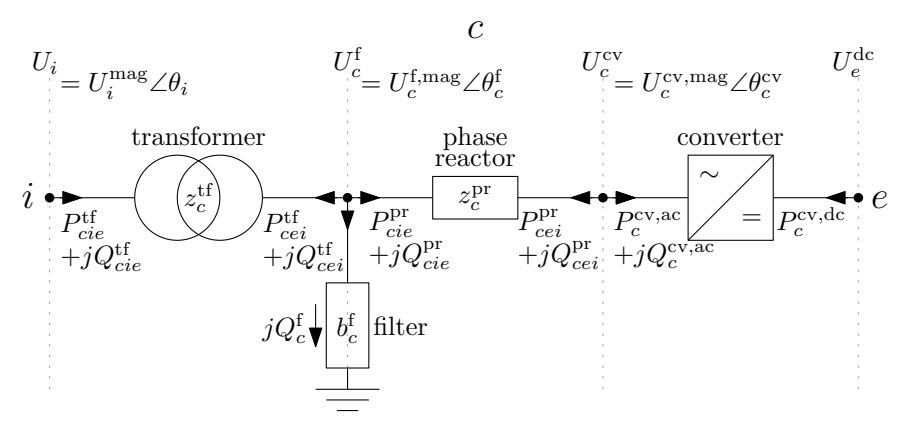

Fig. 2. Overview of parameters and variables in the converter station model.

1) Transformer: The ac voltage is transformed to a voltage suitable for the dc side voltage of the converter. HVDC converter transformers typically have a short circuit impedance of $10-20 \%$ and an X/R ratio of 30 to 40 [27]-[29]. The transformer is characterised by impedance $z_{c}^{\mathrm{tf}}=r_{c}^{\mathrm{tf}}+j x_{c}^{\mathrm{tf}}$, or equivalently by admittance $y_{c}^{\mathrm{tf}}=g_{c}^{\mathrm{tf}}+j b_{c}^{\mathrm{tf}}$. Because the tap setting of the converter transformer can have a significant impact on the voltage control performance of HVDC stations [18], [25], the voltage magnitude transformation factor (tap) is $t_{c}(\mathrm{~V} / \mathrm{V})$ is included. The power flow through the transformer therefore is, in the direction $i \rightarrow e$

$$
\begin{aligned}
P_{c i e}^{\mathrm{tf}} & =g_{c}^{\mathrm{tf}}\left(\frac{U_{i}^{\mathrm{mag}}}{t_{c}}\right)^{2}-g_{c}^{\mathrm{tf}} \frac{U_{i}^{\mathrm{mag}}}{t_{c}} U_{c}^{\mathrm{f}, \mathrm{mag}} \cos \left(\theta_{i}-\theta_{c}^{\mathrm{f}}\right) \\
& -b_{c}^{\mathrm{tf}} \frac{U_{i}^{\mathrm{mag}}}{t_{c}} U_{c}^{\mathrm{f}, \mathrm{mag}} \sin \left(\theta_{i}-\theta_{c}^{\mathrm{f}}\right) \quad \forall c i e \in \mathcal{T}^{\mathrm{cv}}, \\
Q_{c i e}^{\mathrm{tf}} & =-b_{c}^{\mathrm{tf}}\left(\frac{U_{i}^{\mathrm{mag}}}{t_{c}}\right)^{2}+b_{c}^{\mathrm{tf}} \frac{U_{i}^{\mathrm{mag}}}{t_{c}} U_{c}^{\mathrm{f}, \mathrm{mag}} \cos \left(\theta_{i}-\theta_{c}^{\mathrm{f}}\right) \\
& -g_{c}^{\mathrm{tf}} \frac{U_{i}^{\mathrm{mag}}}{t_{c}} U_{c}^{\mathrm{f}, \mathrm{mag}} \sin \left(\theta_{i}-\theta_{c}^{\mathrm{f}}\right) \quad \forall c i e \in \mathcal{T}^{\mathrm{cv}},
\end{aligned}
$$

and in the direction $e \rightarrow i$

$$
\begin{gathered}
P_{c e i}^{\mathrm{tf}}=g_{c}^{\mathrm{tf}}\left(U_{c}^{\mathrm{f}, \mathrm{mag}}\right)^{2}-g_{c}^{\mathrm{tf}} U_{c}^{\mathrm{f}, \mathrm{mag}} \frac{U_{i}^{\mathrm{mag}}}{t_{c}} \cos \left(\theta_{c}^{\mathrm{f}}-\theta_{i}\right) \\
-b_{c}^{\mathrm{tf}} U_{c}^{\mathrm{f}, \mathrm{mag}} \frac{U_{i}^{\mathrm{mag}}}{t_{c}} \sin \left(\theta_{c}^{\mathrm{f}}-\theta_{i}\right) \quad \forall c i e \in \mathcal{T}^{\mathrm{cv}}, \\
Q_{c e i}^{\mathrm{tf}}=-b_{c}^{\mathrm{tf}}\left(U_{c}^{\mathrm{f}, \mathrm{mag}}\right)^{2}+b_{c}^{\mathrm{tf}} U_{c}^{\mathrm{f}, \mathrm{mag}} \frac{U_{i}^{\mathrm{mag}}}{t_{c}} \cos \left(\theta_{c}^{\mathrm{f}}-\theta_{i}\right) \\
-g_{c}^{\mathrm{tf}} U_{c}^{\mathrm{f}, \mathrm{mag}} \frac{U_{i}^{\mathrm{mag}}}{t_{c}} \sin \left(\theta_{c}^{\mathrm{f}}-\theta_{i}\right) \quad \forall c i e \in \mathcal{T}^{\mathrm{cv}} .
\end{gathered}
$$

If the transformer is absent, or if $z_{c}^{\mathrm{tf}} \approx 0$ numerically, the equations for a lossless component are used:

$$
\begin{gathered}
P_{c i e}^{\mathrm{tf}}+P_{c e i}^{\mathrm{tf}}=0 \quad \forall c i e \in \mathcal{T}^{\mathrm{cv}} \\
Q_{c i e}^{\mathrm{tf}}+Q_{c e i}^{\mathrm{tf}}=0 \quad \forall c i e \in \mathcal{T}^{\mathrm{cv}} \\
U_{i}^{\mathrm{mag}}=U_{c}^{\mathrm{f}, \mathrm{mag}} \quad \forall c i e \in \mathcal{T}^{\mathrm{cv}}, \\
\theta_{c}^{\mathrm{f}}=\theta_{i} \quad \forall c i e \in \mathcal{T}^{\mathrm{cv}} .
\end{gathered}
$$


2) Filter: To filter harmonics, filters are often used at HVDC converter stations, especially for the LCC technology. The shunt capacitor has susceptance $b_{c}^{\mathrm{f}}$. The reactive power of the filter capacitor is

$$
Q_{c}^{\mathrm{f}}=-b_{c}^{\mathrm{f}}\left(U_{c}^{\mathrm{f}, \mathrm{mag}}\right)^{2} \quad \forall c i e \in \mathcal{T}^{\mathrm{cv}} .
$$

The nodal balance between the filter capacitor, phase reactor and the transformer is

$$
\begin{gathered}
P_{c i e}^{\mathrm{pr}}+P_{c e i}^{\mathrm{tf}}=0 \quad \forall c i e \in \mathcal{T}^{\mathrm{cv}}, \\
Q_{c i e}^{\mathrm{pr}}+Q_{c e i}^{\mathrm{tf}}+Q_{c}^{\mathrm{f}}=0 \quad \forall c i e \in \mathcal{T}^{\mathrm{cv}} .
\end{gathered}
$$

3) Phase reactor: The inductive part of the filter, the phase reactor impedance is $z_{c}^{\mathrm{pr}}=r_{c}^{\mathrm{pr}}+j x_{c}^{\mathrm{pr}}$, or in admittance form $y_{c}^{\mathrm{pr}}=\frac{1}{z_{c}^{\mathrm{pr}}}=g_{c}^{\mathrm{pr}}+j b_{c}^{\mathrm{pr}}$. As the filter can be represented as a transformer with $t_{c}=1$, the equations (9) - (16) can be applied to represent both the lossy and lossless reactor, respectively. Typically phase reactors have a short circuit impedance of $7.5 \%$ and an $\mathrm{X} / \mathrm{R}$ ratio of 30 [29].

4) $A C / D C$ Converter: The ac side of the converter can inject active and reactive power into the phase reactor. As such, the exchange of power between the ac and dc grid will be modeled by two variables $P_{c}^{\mathrm{cv}, \mathrm{ac}}$ and $Q_{c}^{\mathrm{cv}, \mathrm{ac}}$ defined at the ac bus of all converters. The converter power limits must be respected by the active and reactive power variables:

$$
\begin{gathered}
P_{c}^{\mathrm{cv}, \mathrm{ac}, \mathrm{min}} \leq P_{c}^{\mathrm{cv}, \mathrm{ac}} \leq P_{c}^{\mathrm{cv}, \mathrm{ac}, \mathrm{max}} \quad \forall \text { cie } \in \mathcal{T}^{\mathrm{cv}}, \\
Q_{c}^{\mathrm{cv}, \mathrm{ac}, \mathrm{min}} \leq Q_{c}^{\mathrm{cv}, \mathrm{ac}} \leq Q_{c}^{\mathrm{cv}, \mathrm{ac}, \mathrm{max}} \quad \forall \text { cie } \in \mathcal{T}^{\mathrm{cv}}, \\
\left(P_{c}^{\mathrm{cv}, \mathrm{ac}}\right)^{2}+\left(Q_{c}^{\mathrm{cv}, \mathrm{ac}}\right)^{2} \leq\left(S_{c}^{\mathrm{cv}, \mathrm{ac}, \mathrm{rated}}\right)^{2} \quad \forall c i e \in \mathcal{T}^{\mathrm{cv}} .
\end{gathered}
$$

The converters consume (or inject) a certain amount of active power from the dc grid, modeled by the variable $P_{c}^{\mathrm{c} v \text {,dc }}$ defined at the dc side of each converter. The converters need to respect the dc side active power limits:

$$
P_{c}^{\mathrm{cv}, \mathrm{dc}, \mathrm{min}} \leq P_{c}^{\mathrm{cv}, \mathrm{dc}} \leq P_{c}^{\mathrm{cv}, \mathrm{dc}, \max } \quad \forall \text { cie } \in \mathcal{T}^{\mathrm{cv}} .
$$

The ac and dc side of the converter are linked, through

$$
P_{c}^{\mathrm{cv}, \mathrm{ac}}+P_{c}^{\mathrm{cv}, \mathrm{dc}}=P_{c}^{\mathrm{cv}, \text { loss }} \quad \forall c i e \in \mathcal{T}^{\mathrm{cv}} .
$$

The converter losses themselves are defined with a parameterized loss model,

$$
P_{c}^{\mathrm{cv}, \text { loss }}=a_{c}^{\mathrm{cv}}+b_{c}^{\mathrm{cv}} \cdot I_{c}^{\mathrm{cv}, \mathrm{mag}}+c_{c}^{\mathrm{cv}} \cdot\left(I_{c}^{\mathrm{cv}, \mathrm{mag}}\right)^{2} \forall c i e \in \mathcal{T}^{\mathrm{cv}},
$$

with $a_{c}^{\mathrm{cv}} \geq 0(\mathrm{~W}), b_{c}^{\mathrm{cv}} \geq 0(\mathrm{~W} / \mathrm{A}), c_{c}^{\mathrm{cv}} \geq 0(\Omega)$. $a_{c}^{\mathrm{cv}}$ represents the no load losses of transformers and averaged axillary equipment losses, $b_{c}^{\mathrm{cv}}$ represents the switching losses of valves and freewheeling diodes, whereas $c_{c}^{\mathrm{cv}}$ represents the conduction losses of the valves. Typical values of $a_{c}^{\mathrm{cv}}=11.033 \cdot 10^{-3} \mathrm{pu}$, $b_{c}^{\mathrm{cv}}=3.464 \cdot 10^{-3} \mathrm{pu}, c_{c}^{\mathrm{cv}}=4.4 \cdot 10^{-3} \mathrm{pu}$ for the rectifying and $c_{c}^{\mathrm{cv}}=6.6 \cdot 10^{-3} \mathrm{pu}$ for the inverting converter have been used in the literature [30], [31]. Note that in an implementation, (25) is substituted into (24). Furthermore, $I_{c}^{\mathrm{cv}, \mathrm{mag}}$ is the magnitude of the ac side current of the converter [6]. The ac side current is defined via the active and reactive power injection of the converter as well as the ac side voltage of the converter as

$$
\begin{gathered}
\left(P_{c}^{\mathrm{cv}, \mathrm{ac}}\right)^{2}+\left(Q_{c}^{\mathrm{cv}, \mathrm{ac}}\right)^{2}=\left(U_{c}^{\mathrm{cv}, \mathrm{mag}}\right)^{2}\left(I_{c}^{\mathrm{cv}, \mathrm{mag}}\right)^{2} \forall c i e \in \mathcal{T}^{\mathrm{cv}}, \\
I_{c}^{\mathrm{cv}, \mathrm{mag}} \leq I_{c}^{\mathrm{cv}, \text { rated }} \quad \forall c i e \in \mathcal{T}^{\mathrm{cv}}, \\
U_{c}^{\mathrm{cv}, \mathrm{min}} \leq U_{c}^{\mathrm{cv}, \mathrm{mag}} \leq U_{c}^{\mathrm{cv}, \max } \quad \forall c i e \in \mathcal{T}^{\mathrm{cv}}
\end{gathered}
$$

where $U_{c}^{\mathrm{cv}, \mathrm{mag}}$ is the voltage magnitude at the ac bus of the converter. Finally, the dc converter current is subject to operational limits through

$$
\begin{gathered}
P_{c}^{\mathrm{cv}, \mathrm{dc}}=U_{e}^{\mathrm{dc}} \cdot I_{c}^{\mathrm{cv}, \mathrm{dc}, \mathrm{mag}} \quad \forall c i e \in \mathcal{T}^{\mathrm{cv}}, \\
I_{c}^{\mathrm{cv}, \mathrm{dc}, \mathrm{min}} \leq I_{c}^{\mathrm{cv}, \mathrm{dc}, \mathrm{mag}} \leq I_{c}^{\mathrm{cv}, \mathrm{dc}, \max } \quad \forall c i e \in \mathcal{T}^{\mathrm{cv}} .
\end{gathered}
$$

In this paper a general representation of an converter station model including converter transformer, phase reactor and filters is used. In the implemented ac/dc optimal power flow tool these converter auxiliaries are included in a parametrized way. For instance, LCC converters which use thyristors as power electronic switches, require large harmonic filters as well as reactors in order to avoid large currents during commutation. Two or three level VSC converters use IGBT switches which are controlled via a PWM at frequencies around $1 \mathrm{kHz}$. As such, this type of converters use harmonic filters which are much smaller in comparison to LCCs. MMC type of converters use a high number (a few hundred) of IGBTs connected in series which are switched on or off with a low frequency in order to approximate the sine wave with a step function. This causes a much lower harmonic distortion on the ac voltage and as such, harmonic filters can be omitted. As mentioned above, to accurately model line commutated converters (LCC), the transformers, filters and phase reactors should not be omitted. Note that for LCCs there is a fixed ratio between active and reactive power, i.e.

$$
\begin{array}{ll}
P_{c}^{\mathrm{cv}, \mathrm{ac}}=\cos \varphi_{c} \cdot S_{c}^{\mathrm{cv}, \mathrm{ac}, \text { rated }} & \forall c i e \in \mathcal{T}^{\mathrm{cv}}, \\
Q_{c}^{\mathrm{cv}, \mathrm{ac}}=\sin \varphi_{c} \cdot S_{c}^{\mathrm{cv}, \mathrm{ac}, \text { rated }} & \forall c i e \in \mathcal{T}^{\mathrm{cv}},
\end{array}
$$

where $\varphi_{c}$ is the variable representing the firing angle of the thyristors with $0 \leq \varphi_{c}^{\min } \leq \varphi_{c} \leq \varphi_{c}^{\max } \leq \pi$ [32].

Table II shows an indicative set of parameters for LCC, VSC and MMC type of converters. Finally, it is noted that it is possible to develop case studies with multi-terminal LCC systems, however, this may not be implementable in practice due to control-related challenges.

TABLE II

PARAMETRIZATION OF HVDC CONVERTER MODELS

\begin{tabular}{lcccc}
\hline & transformer & filter & phase reactor & $Q_{c}^{\mathrm{cv}, a c, m i n}$ \\
\hline MMC & $\{0,1\}$ & 0 & $\{0,1\}$ & $\geq-S_{c}^{\mathrm{cv}, \text { ac,rated }}$ \\
VSC & $\{0,1\}$ & 1 & $\{0,1\}$ & $\geq-S_{c}^{\mathrm{cv}, a c, \text { rated }}$ \\
LCC & 1 & 1 & 1 & $\geq 0$ \\
\hline
\end{tabular}

\section{AC and DC Nodal Balance Equations}

Kirchhoff's current law for dc nodes is modeled as

$$
\sum_{c i e \in \mathcal{T}^{\mathrm{cv}}} P_{c}^{\mathrm{cv}, \mathrm{dc}}+\sum_{d e f \in \mathcal{T}^{\mathrm{dc}}} P_{d e f}^{\mathrm{dc}}=-\sum_{m e \in \mathcal{T}^{\text {load,dc }}} P_{m} \forall e \in \mathcal{E},(33)
$$

where $P_{c}^{\mathrm{cv}, \mathrm{dc}}$ are the power injections from converters connected to dc node $e$ and $P_{d e f}^{\mathrm{dc}}$ is the power flow on the dc 
branches connected to dc node $e$. At the ac side, the nodal balance equation is adapted:

$$
\begin{aligned}
& \sum_{c i e \in \mathcal{T}^{\mathrm{cv}}} P_{c i e}^{\mathrm{tf}}+\sum_{l i j \in \mathcal{T}^{\mathrm{ac}}} P_{l i j}^{\mathrm{ac}} \\
& =\sum_{g i \in \mathcal{T}_{\text {gen,ac }}} P_{g}-\sum_{m i \in \mathcal{T}^{\text {load,ac }}} P_{m}-g_{i}^{\text {shunt }}\left(U_{i}^{\text {mag }}\right)^{2} \quad \forall i \in \mathcal{I} \text {, } \\
& \sum_{c i e \in \mathcal{T}^{\mathrm{cv}}} Q_{c i e}^{\mathrm{ff}}+\sum_{l i j \in \mathcal{T}^{\mathrm{ac}}} Q_{l i j}^{\mathrm{ac}} \\
& =\sum_{g i \in \mathcal{T}_{\text {gen,ac }}} Q_{g}-\sum_{m i \in \mathcal{T}^{\text {load,ac }}} Q_{m}+b_{i}^{\text {shunt }}\left(U_{i}^{\text {mag }}\right)^{2} \quad \forall i \in \mathcal{I} \text {, }
\end{aligned}
$$

where $P_{g}+j Q_{g}$ represents a generator's output, $P_{m}+j Q_{m}$ a load's consumption and $g_{i}^{\text {shunt }}+j b_{i}^{\text {shunt }}$ is the ac bus shunt admittance.

\section{Convex Relaxation of AC/DC OPF}

\section{A. Static DC Grid Model}

In this section distinct BIM and BFM formulations are developed which have the same theoretical properties. The symbol $\rightarrow$ is used to indicate substitution w.r.t. the non-linear reference formulation.

1) Bus Injection Model: After substitutions

$$
\left(U_{e}^{\mathrm{dc}}\right)^{2} \rightarrow W_{e}^{\mathrm{dc}},\left(U_{f}^{\mathrm{dc}}\right)^{2} \rightarrow W_{f}^{\mathrm{dc}}, U_{e}^{\mathrm{dc}} U_{f}^{\mathrm{dc}} \rightarrow W_{e f}^{\mathrm{dc}},
$$

and convex relaxation of the voltage cross-products in the spirit of the SOC ac BIM formulation (8), a convex model is obtained:

$$
\begin{gathered}
P_{d e f}^{\mathrm{dc}}=p_{d} \cdot g_{d}^{\mathrm{s}} \cdot\left(W_{e}^{\mathrm{dc}}-W_{e f}^{\mathrm{dc}}\right) \quad \forall \operatorname{def} \in \mathcal{T}^{\mathrm{dc}} \cup \mathcal{T}^{\mathrm{dc}, \mathrm{rev}}, \\
\left(W_{e f}^{\mathrm{dc}}\right)^{2} \leq W_{e}^{\mathrm{dc}} W_{f}^{\mathrm{dc}} \quad \forall \operatorname{def} \in \mathcal{T}^{\mathrm{dc}}
\end{gathered}
$$

2) Branch Flow Model: After substitutions

$$
\left(U_{e}^{\mathrm{dc}}\right)^{2} \rightarrow W_{e}^{\mathrm{dc}},\left(I_{d e f}^{\mathrm{dc}}\right)^{2} \rightarrow i_{d e f}^{\mathrm{sq}, \mathrm{dc}}
$$

and applying the reformulation techniques underlying the SOC BFM ac formulation to (6) - (7), the convex model is obtained:

$$
\begin{gathered}
P_{d}^{\mathrm{dc}, \text { loss }}=p_{d} \cdot r_{d} \cdot i_{d e f}^{\mathrm{sq}, \mathrm{dc}} \quad \forall d e f \in \mathcal{T}^{\mathrm{dc}} \\
\left(P_{d e f}^{\mathrm{dc}}\right)^{2} \leq\left(p_{d}\right)^{2} \cdot W_{e}^{\mathrm{dc}} \cdot i_{d e f}^{\mathrm{sq}, \mathrm{dc}} \quad \forall d e f \in \mathcal{T}^{\mathrm{dc}} \\
W_{f}^{\mathrm{dc}}=W_{e}^{\mathrm{dc}}-2 r_{d} \frac{P_{d e f}^{\mathrm{dc}}}{p_{d}}+\left(r_{d}\right)^{2} i_{d e f}^{\mathrm{sq}, \mathrm{dc}} \forall \operatorname{def} \in \mathcal{T}^{\mathrm{dc}}
\end{gathered}
$$

Because $W_{e}^{\mathrm{dc}}$ and $i_{d e f}^{\mathrm{sq}, \mathrm{dc}}$ are nonnegative, the expression (39) is a rotated second order cone, which makes the inequality convex by construction. It can easily be seen by writing it as a normal second-order cone:

$$
\left\|\left[\begin{array}{c}
\frac{2 P_{d e f}^{\mathrm{dc}}}{p_{d}} \\
W_{e}^{\mathrm{dc}}-i_{d e f}^{\mathrm{sq}, \mathrm{dc}}
\end{array}\right]\right\|_{2} \leq W_{e}^{\mathrm{dc}}+i_{d e f}^{\mathrm{sq}, \mathrm{dc}}
$$

\section{B. Static AC/DC Converter Station Model}

1) AC/DC Converter: The converter loss model includes a term both in the magnitude and in the square of the magnitude of the ac-side current. Whereas the squared term is easily dealt with in BFM convex relaxation formulations, the linear term poses an interesting challenge. Two variables representing the converter current are defined, one representing the normal current magnitude, $I_{c}^{\text {lin,cv,mag }}$, and another representing its square, $i_{c}^{\mathrm{sq}, \mathrm{cv}, \mathrm{mag}}$ :

$$
\begin{gathered}
I_{c}^{\mathrm{cv}, \mathrm{mag}} \rightarrow I_{c}^{\mathrm{lin}, \mathrm{cv}, \mathrm{mag}},\left(I_{c}^{\mathrm{cv}, \mathrm{mag}}\right)^{2} \rightarrow i_{c}^{\mathrm{sq}, \mathrm{cv}, \mathrm{mag}}, \\
I_{c}^{\mathrm{lin}, \mathrm{cv}, \mathrm{mag}} \leq I_{c}^{\mathrm{cv}, \text { rated }} \quad \forall c i e \in \mathcal{T}^{\mathrm{cv}}, \\
i_{c}^{\mathrm{sq}, \mathrm{cv}, \mathrm{mag}} \leq\left(I_{c}^{\mathrm{cv}, \text { rated }}\right)^{2} \quad \forall c i e \in \mathcal{T}^{\mathrm{cv}} .
\end{gathered}
$$

The convex relaxation of $I_{c}^{\mathrm{lin}, \mathrm{cv}, \mathrm{mag}}=\sqrt{i_{c}^{\mathrm{sq}, \mathrm{cv}, \mathrm{mag}}}$ is

$$
\begin{gathered}
\left(I_{c}^{\mathrm{lin}, \mathrm{cv}, \mathrm{mag}}\right)^{2} \leq i_{c}^{\mathrm{sq}, \mathrm{cv}, \mathrm{mag}} \quad \forall c i e \in \mathcal{T}^{\mathrm{cv}}, \\
i_{c}^{\mathrm{sq}, \mathrm{cv}, \mathrm{mag}} \leq I_{c}^{\mathrm{lin}, \mathrm{cv}, \mathrm{mag}} I_{c}^{\mathrm{cv}, \text { rated }} \quad \forall c i e \in \mathcal{T}^{\mathrm{cv}} .
\end{gathered}
$$

The variables $I_{c}^{\text {lin,cv,mag }}$ and $i_{c}^{\text {sq,cv,mag }}$ are furthermore linked through inequalities defined in the $\mathrm{W}$ variable space:

$$
\begin{gathered}
\left(U_{c}^{\mathrm{cv}, \mathrm{mag}}\right)^{2} \rightarrow W_{c}^{\mathrm{cv}}, U_{c}^{\mathrm{cv}, \mathrm{mag}} \leq U_{c}^{\mathrm{cv}, \mathrm{max}} \rightarrow W_{c}^{\mathrm{cv}} \leq\left(U_{c}^{\mathrm{cv}, \mathrm{max}}\right)^{2}, \\
P_{c}^{\mathrm{cv}, \mathrm{loss}}=a_{c}^{\mathrm{cv}}+b_{c}^{\mathrm{cv}} \cdot I_{c}^{\mathrm{lin}, \mathrm{cv}, \mathrm{mag}}+c_{c}^{\mathrm{cv}} \cdot i_{c}^{\mathrm{sq}, \mathrm{cv}, \mathrm{mag}} \forall c i e \in \mathcal{T}^{\mathrm{cv}},(46) \\
\left(P_{c}^{\mathrm{cv}, \mathrm{ac}}\right)^{2}+\left(Q_{c}^{\mathrm{cv}, \mathrm{ac}}\right)^{2} \leq W_{c}^{\mathrm{cv}} \cdot i_{c}^{\mathrm{sq}, \mathrm{cv}, \mathrm{mag}} \forall c i e \in \mathcal{T}^{\mathrm{cv}} . \quad(47)
\end{gathered}
$$

Finally, using the bound on the voltage magnitude of the converter, a valid inequality is obtained:

$$
\left(P_{c}^{\mathrm{cv}, \mathrm{ac}}\right)^{2}+\left(Q_{c}^{\mathrm{cv}, \mathrm{ac}}\right)^{2} \leq\left(U_{c}^{\mathrm{cv}, \mathrm{max}}\right)^{2}\left(I_{c}^{\mathrm{lin}, \mathrm{cv}, \mathrm{mag}}\right)^{2} \forall c i e \in \mathcal{T}^{\mathrm{cv}}
$$

If $b_{c}^{\mathrm{cv}}=0$, the equations obtained are similar to those of the classic BFM SOC relaxation, but without linking the voltages from the ac to the $\mathrm{dc}$ side. Nevertheless, if $b_{c}^{\mathrm{cv}} \neq 0$, a supplemental relaxation gap may be observed. In the optimum the idea is that the error is negligible, i.e. $I_{c}^{\mathrm{cv}, \mathrm{mag}} \approx I_{c}^{\mathrm{lin}, \mathrm{cv}, \mathrm{mag}} \approx$ $\sqrt{i_{c}^{\mathrm{sq}, \mathrm{cv}, \mathrm{mag}}}$.

To develop a convex relaxation formulation of the LCC model, we first derive four parameters for each converter, based on the bounds of $\varphi_{c}$ :

$$
\begin{aligned}
P_{c}^{1} & =\cos \varphi_{c}^{\min } \cdot S_{c}^{\mathrm{cv}, \mathrm{ac}, \text { rated }} \\
P_{c}^{2} & =\cos \varphi_{c}^{\max } \cdot S_{c}^{\mathrm{cv}, \mathrm{ac}, \text { rated }} \\
Q_{c}^{1} & =\sin \varphi_{c}^{\min } \cdot S_{c}^{\mathrm{cv}, \mathrm{ac}, \text { rated }} \\
Q_{c}^{2} & =\sin \varphi_{c}^{\max } \cdot S_{c}^{\mathrm{cv}, \mathrm{ac}, \text { rated }} .
\end{aligned}
$$

These then are used to define a lower bound on the reactive power:

$$
Q_{c}^{\mathrm{cv}, \mathrm{ac}} \geq Q_{c}^{1}+\left(P_{c}^{\mathrm{cv}, \mathrm{ac}}-P_{c}^{1}\right) \frac{\left(Q_{c}^{2}-Q_{c}^{1}\right)}{\left(P_{c}^{2}-P_{c}^{1}\right)} \forall c i e \in \mathcal{T}^{\mathrm{cv}}
$$

Note that for $0 \leq \varphi_{c}^{\min } \leq \varphi_{c}^{\max } \leq \pi$, this implies $Q_{c}^{\mathrm{cv}, \mathrm{ac}} \geq 0$, thereby always behaving inductively with respect to the ac grid. The tightness of convex relaxation will be better when $\varphi_{c}^{\min } \approx \varphi_{c}^{\max }$. Simply defining the operation mode, i.e. $\varphi_{c}^{\max } \leq$ $\frac{\pi}{2}$ (rectifier) or $\varphi_{c}^{\min } \geq \frac{\pi}{2}$ (inverter) already significantly improves accuracy. 
2) Phase Reactor BIM: Applying the substitutions

$$
\begin{gathered}
\left(U_{c}^{\mathrm{f}, \mathrm{mag}}\right)^{2} \rightarrow W_{c}^{\mathrm{f}},\left(U_{c}^{\mathrm{cv}, \mathrm{mag}}\right)^{2} \rightarrow W_{c}^{\mathrm{cv}}, \\
U_{c}^{\mathrm{f}, \mathrm{mag}} U_{c}^{\mathrm{cv}, \mathrm{mag}} \cos \left(\theta_{c}^{\mathrm{f}}-\theta_{c}^{\mathrm{cv}}\right) \rightarrow R_{c}^{\mathrm{pr}}, \\
U_{c}^{\mathrm{f}, \mathrm{mag}} U_{c}^{\mathrm{cv}, \mathrm{mag}} \sin \left(\theta_{c}^{\mathrm{f}}-\theta_{c}^{\mathrm{cv}}\right) \rightarrow T_{c}^{\mathrm{pr}},
\end{gathered}
$$

the classic SOC BIM formulation is obtained:

$$
\begin{gathered}
\left(R_{c}^{\mathrm{pr}}\right)^{2}+\left(T_{c}^{\mathrm{pr}}\right)^{2} \leq W_{c}^{\mathrm{f}} W_{c}^{\mathrm{cr}} \quad \forall c i e \in \mathcal{T}^{\mathrm{cv}}, \\
P_{c i e}^{\mathrm{pr}}=g_{c}^{\mathrm{pr}} W_{c}^{\mathrm{f}}-g_{c}^{\mathrm{pr}} R_{c}^{\mathrm{pr}}-b_{c}^{\mathrm{pr}} T_{c}^{\mathrm{pr}} \forall c i e \in \mathcal{T}^{\mathrm{cv}}, \\
Q_{c i e}^{\mathrm{pr}}=-b_{c}^{\mathrm{pr}} W_{c}^{\mathrm{f}}+b_{c}^{\mathrm{pr}} R_{c}^{\mathrm{pr}}-g_{c}^{\mathrm{pr}} T_{c}^{\mathrm{pr}} \forall c i e \in \mathcal{T}^{\mathrm{cv}}, \\
P_{c e i}^{\mathrm{pr}}=g_{c}^{\mathrm{pr}} W_{c}^{\mathrm{cv}}-g_{c}^{\mathrm{pr}} R_{c}^{\mathrm{pr}}-b_{c}^{\mathrm{pr}}\left(-T_{c}^{\mathrm{pr}}\right) \quad \forall c i e \in \mathcal{T}^{\mathrm{cv}}, \\
Q_{c e i}^{\mathrm{pr}}=-b_{c}^{\mathrm{pr}} W_{c}^{\mathrm{cv}}+b_{c}^{\mathrm{pr}} R_{c}^{\mathrm{pr}}-g_{c}^{\mathrm{pr}}\left(-T_{c}^{\mathrm{pr}}\right) \quad \forall c i e \in \mathcal{T}^{\mathrm{cv}} .
\end{gathered}
$$

If the phase reactor is absent, or if $z_{c}^{\mathrm{pr}} \approx 0$ numerically, the following equations, defining a lossless component, are used instead:

$$
\begin{gathered}
P_{c i e}^{\mathrm{pr}}+P_{c e i}^{\mathrm{pr}}=0 \quad \forall c i e \in \mathcal{T}^{\mathrm{cv}} \\
Q_{c i e}^{\mathrm{pr}}+Q_{c e i}^{\mathrm{pr}}=0 \quad \forall c i e \in \mathcal{T}^{\mathrm{cv}} \\
R_{c}^{\mathrm{pr}}=W_{c}^{\mathrm{f}}=W_{c}^{\mathrm{cv}} \quad \forall c i e \in \mathcal{T}^{\mathrm{cv}} \\
T_{c}^{\mathrm{pr}}=0 \quad \forall c i e \in \mathcal{T}^{\mathrm{cv}} .
\end{gathered}
$$

3) Phase Reactor BFM: Applying substitutions

$$
\begin{gathered}
\left(U_{c}^{\mathrm{f}, \mathrm{mag}}\right)^{2} \rightarrow W_{c}^{\mathrm{f}},\left(U_{c}^{\mathrm{cv}, \mathrm{mag}}\right)^{2} \rightarrow W_{c}^{\mathrm{cv}} \\
\left|I_{c}^{\mathrm{pr}}\right|^{2} \rightarrow i_{c}^{\mathrm{sq}, \mathrm{pr}},
\end{gathered}
$$

the classic SOC BFM formulation is obtained:

$$
\begin{gathered}
\left(P_{c i e}^{\mathrm{pr}}\right)^{2}+\left(Q_{c i e}^{\mathrm{pr}}\right)^{2} \leq W_{c}^{\mathrm{f}} i_{c}^{\mathrm{sq}, \mathrm{pr}} \forall c i e \in \mathcal{T}^{\mathrm{cv}}, \\
P_{c i e}^{\mathrm{pr}}+P_{c e i}^{\mathrm{pr}}=r_{c}^{\mathrm{pr}} i_{c}^{\mathrm{sq}, \mathrm{pr}} \forall c i e \in \mathcal{T}^{\mathrm{cv}} \\
Q_{c i e}^{\mathrm{pr}}+Q_{c e i}^{\mathrm{pr}}=x_{c}^{\mathrm{pr}} i_{c}^{\mathrm{sq}, \mathrm{pr}} \forall c i e \in \mathcal{T}^{\mathrm{cv}} \\
W_{c}^{\mathrm{cv}}=W_{c}^{\mathrm{f}}-2\left(r_{c}^{\mathrm{pr}} P_{c i e}^{\mathrm{pr}}+x_{c}^{\mathrm{pr}} Q_{c i e}^{\mathrm{pr}}\right) \\
+\left(\left(r_{c}^{\mathrm{pr}}\right)^{2}+\left(x_{c}^{\mathrm{pr}}\right)^{2}\right) i_{c}^{\mathrm{sq}, \mathrm{pr}} \forall c i e \in \mathcal{T}^{\mathrm{cv}} .
\end{gathered}
$$

4) Filter (BFM and BIM): The reactive power of the filter capacitor in the new voltage variables is

$$
Q_{c}^{\mathrm{f}}=-b_{c}^{\mathrm{f}} W_{c}^{\mathrm{f}} \quad \forall c i e \in \mathcal{T}^{\mathrm{cv}} .
$$

The nodal balance between the filter capacitor, phase reactor and the transformer remains unchanged, i.e. (18) - (19).

5) Transformer BIM: Applying substitutions

$$
\begin{gathered}
\left(U_{i}^{\mathrm{mag}}\right)^{2} \rightarrow W_{i},\left(U_{c}^{\mathrm{f}, \mathrm{mag}}\right)^{2} \rightarrow W_{c}^{\mathrm{f}}, \\
U_{i}^{\mathrm{mag}} U_{c}^{\mathrm{f}, \mathrm{mag}} \cos \left(\theta_{i}-\theta_{c}^{\mathrm{f}}\right) \rightarrow R_{i c}^{\mathrm{tf}}, \\
U_{i}^{\mathrm{mag}} U_{c}^{\mathrm{f}, \mathrm{mag}} \sin \left(\theta_{i}-\theta_{c}^{\mathrm{f}}\right) \rightarrow T_{i c}^{\mathrm{ff}},
\end{gathered}
$$

the classic SOC BIM formulation is obtained:

$$
\begin{gathered}
\left(R_{i c}^{\mathrm{tf}}\right)^{2}+\left(T_{i c}^{\mathrm{tf}}\right)^{2} \leq W_{i} W_{c}^{\mathrm{f}} \forall c i e \in \mathcal{T}^{\mathrm{cv}}, \\
P_{c i e}^{\mathrm{tf}}=g_{c}^{\mathrm{tf}} \frac{W_{i}}{\left(t_{c}\right)^{2}}-g_{c}^{\mathrm{tf}} \frac{R_{i c}^{\mathrm{tf}}}{t_{c}}-b_{c}^{\mathrm{tf}} \frac{T_{i c}^{\mathrm{tf}}}{t_{c}} \quad \forall c i e \in \mathcal{T}^{\mathrm{cv}}, \\
Q_{c i e}^{\mathrm{tf}}=-b_{c}^{\mathrm{tf}} \frac{W_{i}}{\left(t_{c}\right)^{2}}+b_{c}^{\mathrm{tf}} \frac{R_{i c}^{\mathrm{tf}}}{t_{c}}-g_{c}^{\mathrm{tf}} \frac{T_{i c}^{\mathrm{tf}}}{t_{c}} \quad \forall c i e \in \mathcal{T}^{\mathrm{cv}}, \\
P_{c e i}^{\mathrm{tf}}=g_{c}^{\mathrm{tf}} W_{c}^{\mathrm{f}}-g_{c}^{\mathrm{tf}} \frac{R_{i c}^{\mathrm{tf}}}{t_{c}}-b_{c}^{\mathrm{tf}}\left(-\frac{T_{i c}^{\mathrm{ff}}}{t_{c}}\right) \quad \forall c i e \in \mathcal{T}^{\mathrm{cv}}, \\
Q_{c e i}^{\mathrm{tf}}=-b_{c}^{\mathrm{tf}} W_{c}^{\mathrm{f}}+b_{c}^{\mathrm{tf}} \frac{R_{i c}^{\mathrm{tf}}}{t_{c}}-g_{c}^{\mathrm{tf}}\left(-\frac{T_{i c}^{\mathrm{tf}}}{t_{c}}\right) \quad \forall c i e \in \mathcal{T}^{\mathrm{cv}} .
\end{gathered}
$$

If the transformer is absent, or if $z_{c}^{\mathrm{tf}} \approx 0$ numerically, the equations for a lossless component are used:

$$
\begin{gathered}
P_{c i e}^{\mathrm{tf}}+P_{c e i}^{\mathrm{tf}}=0 \quad \forall c i e \in \mathcal{T}^{\mathrm{cv}}, \\
Q_{c i e}^{\mathrm{tf}}+Q_{c e i}^{\mathrm{tf}}=0 \quad \forall c i e \in \mathcal{T}^{\mathrm{cv}}, \\
\frac{W_{i}}{\left(t_{c}\right)^{2}}=W_{c}^{\mathrm{f}}=R_{i c}^{\mathrm{tf}} \quad \forall c i e \in \mathcal{T}^{\mathrm{cv}} \\
T_{i c}^{\mathrm{tf}}=0 \quad \forall c i e \in \mathcal{T}^{\mathrm{cv}} .
\end{gathered}
$$

6) Transformer BFM: Applying substitutions

$$
\begin{gathered}
\left(U_{i}^{\mathrm{mag}}\right)^{2} \rightarrow W_{i}, \quad\left(U_{c}^{\mathrm{f}, \mathrm{mag}}\right)^{2} \rightarrow W_{c}^{\mathrm{f}} \\
\left|I_{c}^{\mathrm{tf}}\right|^{2} \rightarrow i_{c}^{\mathrm{sq}, \mathrm{ff}},
\end{gathered}
$$

the classic SOC BFM formulation is obtained:

$$
\begin{gathered}
\left(P_{c i e}^{\mathrm{tf}}\right)^{2}+\left(Q_{c i e}^{\mathrm{tf}}\right)^{2} \leq \frac{W_{i}}{\left(t_{c}\right)^{2}} i_{c}^{\mathrm{sq}, \mathrm{tf}} \forall c i e \in \mathcal{T}^{\mathrm{cv}}, \\
P_{c i e}^{\mathrm{tf}}+P_{c e i}^{\mathrm{tf}}=r_{c}^{\mathrm{tf}} i_{c}^{\mathrm{sq}, \mathrm{tf}} \forall c i e \in \mathcal{T}^{\mathrm{cv}} \\
Q_{c i e}^{\mathrm{tf}}+Q_{c e i}^{\mathrm{tf}}=x_{c}^{\mathrm{tf}} i_{c}^{\mathrm{sq}, \mathrm{tf}} \forall c i e \in \mathcal{T}^{\mathrm{cv}}, \\
W_{c}^{\mathrm{f}}=\frac{W_{i}}{\left(t_{c}\right)^{2}}-2\left(r_{c}^{\mathrm{tf}} P_{c i e}^{\mathrm{tf}}+x_{c}^{\mathrm{tf}} Q_{c i e}^{\mathrm{tf}}\right) \\
+\left(\left(r_{c}^{\mathrm{tf}}\right)^{2}+\left(x_{c}^{\mathrm{tf}}\right)^{2}\right) i_{c}^{\mathrm{sq}, \mathrm{tf}} \forall c i e \in \mathcal{T}^{\mathrm{cv}} .
\end{gathered}
$$

\section{LINEAR APPROXIMATION TO AC/DC OPF}

The same assumptions underlying 'DC' linear approximation to ac power flow equations are applied to the dc power flow equations ${ }^{2}$.

\section{A. Static DC Grid Model}

Under the assumptions $U_{e}^{\mathrm{dc}} \approx 1$ p.u., $r_{d} \approx 0$, the power flow reduces to

$$
P_{d e f}^{\mathrm{dc}}+P_{d f e}^{\mathrm{dc}}=0 \quad \forall d e f \in \mathcal{T}^{\mathrm{dc}}
$$

which is categorized as a network flow (NF) formulation.

\section{B. Static AC/DC Converter Station Model}

1) $A C / D C$ Converter: Under the assumptions $U_{c}^{\mathrm{cv}, \mathrm{mag}}=$ $U \approx 1$ p.u., $c_{c}^{\mathrm{cv}} \approx 0$ and $I_{c}^{\mathrm{cv}, \mathrm{mag}} \approx \frac{P_{c}^{\mathrm{c}, a c}}{U_{c}^{\mathrm{c}, m a g},}$, the power flow through, and losses of, the converter are derived as

$$
P_{c}^{\mathrm{cv}, \mathrm{ac}}+P_{c}^{\mathrm{cv}, \mathrm{dc}}=a_{c}^{\mathrm{cv}}+b_{c}^{\mathrm{cv}} \cdot \frac{P_{c}^{\mathrm{cv}, \mathrm{ac}}}{U} \forall c i e \in \mathcal{T}^{\mathrm{cv}}
$$

which is linear in active power variables. Note that the line losses may be negative when $P_{c}^{\mathrm{cv}, \text { ac }} \leq 0$ and this model reduces to a network flow model for $a_{c}^{\mathrm{cv}}=b_{c}^{\mathrm{cv}}=0$ which is used in Matpower [11].

\footnotetext{
${ }^{2}$ to limit confusion, when referring to dc grids, a lower case $d c$ is used (except in titles), when referring to the 'DC' linear approximation, it is stylized
} in capitals, in quotes. 
2) Phase Reactor: Under the assumptions $U_{i}^{\text {mag }}=$ $U_{c}^{\mathrm{cv}, \mathrm{mag}}=U_{c}^{\mathrm{f}, \mathrm{mag}}=U \approx 1$ p.u., and the small voltage angle condition $\theta \approx 0$, and applying $\cos (\theta) \approx 1$ and $\sin (\theta) \approx \theta$, the power flow through the phase reactor is developed as

$$
\begin{array}{ll}
P_{c i e}^{\mathrm{pr}}=-b_{c}^{\mathrm{pr}} U U\left(\theta_{c}^{\mathrm{f}}-\theta_{c}^{\mathrm{cv}}\right) & \forall c i e \in \mathcal{T}^{\mathrm{cv}}, \\
P_{c e i}^{\mathrm{pr}}=-b_{c}^{\mathrm{pr}} U U\left(\theta_{c}^{\mathrm{cv}}-\theta_{c}^{\mathrm{f}}\right) & \forall c i e \in \mathcal{T}^{\mathrm{cv}} .
\end{array}
$$

The lossless network flow approximation finally is

$$
\begin{gathered}
P_{c i e}^{\mathrm{pr}}+P_{c e i}^{\mathrm{pr}}=0 \quad \forall c i e \in \mathcal{T}^{\mathrm{cv}}, \\
\theta_{c}^{\mathrm{f}}=\theta_{c}^{\mathrm{cv}} \quad \forall c i e \in \mathcal{T}^{\mathrm{cv}} .
\end{gathered}
$$

3) Filter: The nodal balance at the filter, between the phase reactor and the transformer is

$$
P_{c i e}^{\mathrm{pr}}+P_{c e i}^{\mathrm{tf}}=0 \quad \forall c i e \in \mathcal{T}^{\mathrm{cv}}
$$

4) Transformer: Further assuming $U_{i}^{\mathrm{mag}}=U_{c}^{\mathrm{f} \text {,mag }}=$ $U_{c}^{\mathrm{cv}, \mathrm{mag}}=U \approx 1 \mathrm{pu}$, and performing the small angle approximation, the transformer equations become

$$
\begin{aligned}
& P_{c i e}^{\mathrm{tf}}=-b_{c}^{\mathrm{tf}} U \frac{U}{t_{c}}\left(\theta_{i}-\theta_{c}^{\mathrm{f}}\right) \quad \forall c i e \in \mathcal{T}^{\mathrm{cv}}, \\
& P_{c e i}^{\mathrm{tf}}=-b_{c}^{\mathrm{tf}} U \frac{U}{t_{c}}\left(\theta_{c}^{\mathrm{f}}-\theta_{i}\right) \quad \forall c i e \in \mathcal{T}^{\mathrm{cv}} .
\end{aligned}
$$

The lossless network flow approximation finally is

$$
\begin{gathered}
P_{c i e}^{\mathrm{tf}}+P_{c e i}^{\mathrm{tf}}=0 \quad \forall c i e \in \mathcal{T}^{\mathrm{cv}}, \\
\theta_{i}=\theta_{c}^{\mathrm{f}} \quad \forall c i e \in \mathcal{T}^{\mathrm{cv}} .
\end{gathered}
$$

\section{AC/DC OPF Formulation OVERVIEW \& PROPERTIES}

It is assumed that the ac branch model includes an ideal transformer at the from side in series with a $\pi$-element (as in [11]), which is able to represent both lossy lines and lossy transformers. Conversely, dc lines are modeled as a series resistance.

Fig. 3 summarizes the mapping of the de to the ac formulation hierarchy. There exist fewer distinct dc branch formu-

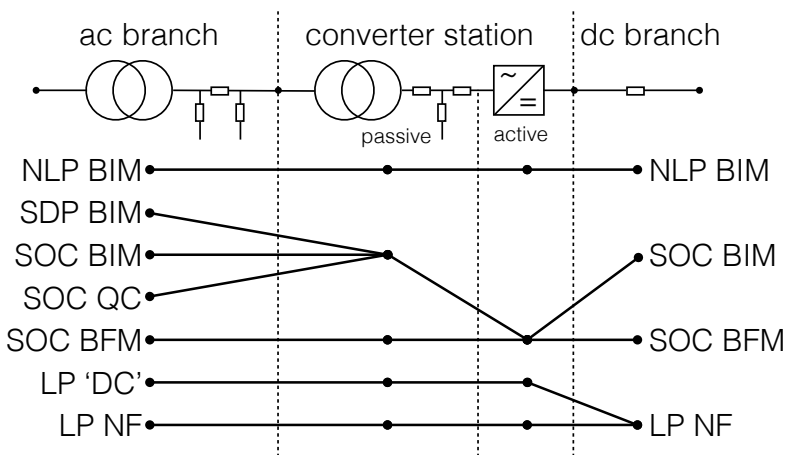

Fig. 3. Mapping of ac, dc and converter formulations: ac formulation names on the left, dc formulation names on the right.

lations (right side of Fig. 3) than there exist ac formulations (left side of Fig. 3). Similar to ac grids, the SOC BIM and $\mathrm{BFM}$ are equivalent for the ac-side passive elements in the converter station, as well as the dc grid [17].

As there are no variables and constraints related to voltage angles in a dc grid, branch voltage magnitude drop equations are sufficient to represent Kirchhoff's current law (KVL) in any circuit, i.e. both in radial and meshed dc grids. Given the properties of the semi-definite programming (SDP) [33], quadratic convex (QC) [34] and linearized 'DC' formulation in ac grids w.r.t. voltage angle variables, it is easy to see why these formulations are missing for dc grids. We note that the relaxation strength results of ac OPF formulations [34] are unaffected by the dc extensions, therefore SDP is incomparable to conventional QC, but both are tighter than the conventional SOC relaxation. Furthermore, given the radial nature of the converter stations' passive components, SOC $\mathrm{BIM}$ is the tightest convex relaxation, therefore possessing the same model properties as SDP and QC in this context.

\section{A. AC/DC NLP-NLP}

An exact NLP formulation exists for all elements. As this bus injection model is nonconvex but continuous, it can be solved to local optimality through the interior-point method for NLP. In this case Ipopt is used [35].

\section{B. AC/DC SDP-SOC, QC-SOC and SOC-SOC}

The SDP, QC and SOC formulations for ac grids all map to the SOC formulation for the converter stations and $\mathrm{dc}$ branches. Therefore, the choice of ac formulation depends on the properties of the ac system being modeled. For instance, if it is radial, the SOC BIM or BFM are the tightest formulations (SDP reduces to SOC) [36]. Note that lifted nonlinear cuts can be applied to these convex ac formulations [37], and that these are implemented in PowerModels.

The SOC dc BFM or BIM are exact for meshed dc grids due to absence of voltage angles and related constraints, per conditions [36]. The converter model is not expected to be exact, but is expected to have properties similar to those of SOC ac BFM formulations when $b_{c}^{\mathrm{cv}} \neq 0$. Ipopt is used to solve the SOC models, but Mosek is used for the SDP models [38].

\section{C. $A C / D C$ ' $D C$ '-NF}

The combination of the 'DC' linear approximation to the ac grid part and network flow approximation to the dc grid part results in a LP model. This model is lossless except for the losses in the converter itself (not the passive components). For this formulation, Ipopt is used as solver.

\section{NUMERICAL EXPERIMENTS}

\section{A. Implementation and Validation}

Results were validated in terms of ac power flow feasibility w.r.t. MatACDC [6], with its extensive convert station models, exact dc line model and support for meshed dc grids. Furthermore, using the 'dcline' model of Matpower ${ }^{3}$, the feasibility and optimality of the ac and linearized 'DC' formulation were verified. Finally, to maintain compatibility with the conventional OPF problem type definition, feasibility and optimality of all formulations, for pure ac grids, were validated w.r.t. all the discussed formulations, which are available in PowerModels [21].

\footnotetext{
${ }^{3}$ which is also supported by PowerModels [21]
} 


\section{B. Benchmark Cases and Results}

Fig. 4 illustrates case5_acdc which is based on the IEEE 5 bus test system and is also used in [24]. A three node dc grid has been embedded in the 5 bus ac system using VSC converters. In case5_lcc, the VSC HVDC converters have been replaced by LCC converters. For case5_2grids two identical case5_acdc grids have been connected via an ac branch. case5_dcgrid consists of one ac bus and a dc grid with four buses including dc loads. It represents a dc system with only one ac generator, to provide power, through a converter, to a dc grid with dc loads. All load is supplied by the generator connected to the ac bus. case5_dc has been used to validate against Matpower, and includes one HVDC link between buses 3 and 5 of the IEEE 5 bus test system. case 5 b2bdc uses a back-to-back HVDC link using the detailed converter model (represented by two converters with an ac bus in between). case24_3zones connects three IEEE 24 bus reliability test systems using a dc grid and is obtained from [6]. The both cases case_39 and case_3120sp_acdc are obtained from [10] and represent dc grid extensions of the IEEE 39 bus test system and the 3210 bus Polish system, respectively. case_588_sdet_acdc is an extension of the 588 bus system obtained from [39]. A seven bus, eight branch dc grid has been embedded within the ac system. Table III illustrates key properties of the test cases.

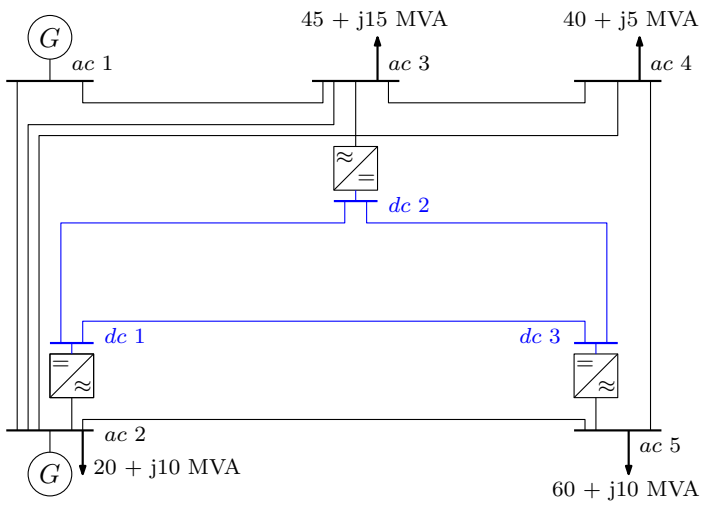

Fig. 4. Single line diagram of case5_acdc. Blue lines indicate dc lines.

TABLE III AC/DC OPF Test CASE Properties

\begin{tabular}{lrrrccc}
\hline Test case & $\begin{array}{r}\text { \#nodes } \\
\text { ac }\end{array}$ & $\begin{array}{r}\text { \#nodes } \\
\text { dc }\end{array}$ & $\begin{array}{r}\text { \#conv } \\
\text { ac/dc }\end{array}$ & $\begin{array}{c}\text { dc } \\
\text { mesh? }\end{array}$ & $\begin{array}{c}\text { \#ac } \\
\text { grids }\end{array}$ & $\begin{array}{c}\text { TF } \\
\text { tap? }\end{array}$ \\
\hline case5_2grids & 10 & 2 & 2 & $\boldsymbol{X}$ & 2 & $\boldsymbol{X}$ \\
case5_acdc & 5 & 3 & 3 & $\checkmark$ & 1 & $\boldsymbol{X}$ \\
case5_lcc & 5 & 3 & 3 & $\checkmark$ & 1 & $\boldsymbol{X}$ \\
case5_dc & 5 & 2 & 2 & $\boldsymbol{X}$ & 1 & $\boldsymbol{X}$ \\
case5_dcgrid & 1 & 4 & 1 & $\checkmark$ & 1 & $\checkmark$ \\
case5_b2bdc & 5 & 1 & 2 & $\boldsymbol{X}$ & 1 & $\boldsymbol{X}$ \\
case24_3zones & 50 & 7 & 7 & $\checkmark$ & 3 & $\boldsymbol{X}$ \\
case_39 & 39 & 10 & 10 & $\checkmark$ & 1 & $\boldsymbol{X}$ \\
case_588_sdet_acdc & 588 & 7 & 7 & $\checkmark$ & 1 & $\boldsymbol{X}$ \\
case_3120sp_acdc & 3120 & 5 & 5 & $\checkmark$ & 1 & $\boldsymbol{X}$ \\
\hline
\end{tabular}

The optimization objective remains the same as in a normal OPF, i.e. the minimization of the generation cost expressed as a polynomial function of its active power output. The gap between a specific formulation and the BIM NLP ac/dc (local) optimum is calculated as

$$
\operatorname{gap}(\text { form })=\frac{\text { objective(BIM NLP) }- \text { objective(form) }}{\text { objective(BIM NLP) }} \text {. }
$$

Table IV summarizes the results of a number of test cases.

It is seen that the SDP formulation is generally tight, but that it is more sensitive to numerical issues. For the developed cases, SOC BIM and SOC BFM results are identical up to numerical accuracy. For the small cases, the QC formulation appears to be identical with SOC BIM and SOC BFM, whereas for larger cases there is considerable difference in the optimality gaps. This suggests a need for developing a greater variety of test cases. Certain test cases show a significant optimality gap for the QC and SOC relaxations, others are tight. It is observed that in unconstrained (i.e. power and current of dc branches and converter stations not at their limits) conditions, the SOC formulation is exact (numerically). As expected from the derivation, inexactness is observed when the converter's loss parameter $b_{c}^{\mathrm{cv}} \neq 0$.

Table V illustrates the computation time of the different formulations. In the analysed test cases we can observe that the SOC BFM formulation is faster than the SOC BIM formulation with very comparable optimality gaps to the SOC BIM formulation. Nevertheless, the results primarily suggest a need for a wider array of test cases to use in benchmarking.

\section{CONCLUSIONS}

In this paper a comprehensive ac/dc OPF model is introduced, and expanded in both the BIM and BFM form in a harmonized choice of symbols for variables and parameters. Consistent dc grid and ac/dc converter formulations, compatible with common power-voltage-variable-based ac OPF formulations, have been derived. The problem definition includes the conventional ac-grid-only OPF as an edge case, through empty sets for dc nodes and converters. The OPF model considers ac grid, ac/dc converter station and dc grid losses and can be used to schedule HVDC converters in both active and reactive power. Converter stations can be parameterized to reflect the behavior of LCC, VSC and MMC converters. It is possible to omit the converter transformer, filter and/or reactor from the model for the representation of different converter types, furthermore improving numerical stability, instead of being forced to use small impedance values. Parallel lines and meshed topologies are supported for both ac and dc grids. Finally, a mapping of common ac OPF formulations to the formulations for ac/dc converter stations and dc grids has been developed.

An open-source implementation of the model is provided [23], extending the existing open-source project 'PowerModels.jl' [21] which can serve as a framework to test the accuracy and speed of existing and newly developed relaxation schemes for the optimal power flow problem for ac/dc grids. This implementation supports multiple asynchronous ac grids and LCC, VSC and MMC dc systems as well as dc grids in a single OPF problem. In terms of power flow feasibility, the implementation is validated w.r.t. MatACDC. All formulations 
TABLE IV

AC/DC OPF OBJECTIVE VALUES AND GAP. (NUMERICAL ISSUE INDICATED *)

\begin{tabular}{|c|c|c|c|c|c|c|c|c|c|c|c|}
\hline \multirow[t]{2}{*}{ case } & \multirow{2}{*}{$\begin{array}{l}\text { BIM NLP } \\
\text { obj }(\$ / h)\end{array}$} & \multicolumn{2}{|l|}{ SDP } & \multicolumn{2}{|l|}{$\mathrm{QC}$} & \multicolumn{2}{|l|}{ BIM SOC } & \multicolumn{2}{|l|}{ BFM SOC } & \multicolumn{2}{|l|}{ 'DC' } \\
\hline & & obj $(\$ / h)$ & gap $(\%)$ & obj $(\$ / h)$ & gap $(\%)$ & obj $(\$ / h)$ & gap $(\%)$ & obj $(\$ / h)$ & gap $(\%)$ & obj $(\$ / h)$ & 'gap' (\%) \\
\hline case5_2grids & 397.367 & 397.366 & 0 & 363.502 & 8.522 & 363.502 & 8.522 & 363.503 & 8.522 & 371.005 & 6.634 \\
\hline case5_acdc & 194.139 & 194.12 & 0.009 & 183.763 & 5.345 & 183.763 & 5.345 & 183.763 & 5.344 & 171.686 & 11.566 \\
\hline case5_lcc & 196.655 & 194.12 & 1.289 & 183.763 & 6.556 & 183.763 & 6.556 & 183.763 & 6.556 & 171.686 & 12.697 \\
\hline case5_dc & 17762.4 & 16654.9 & 6.235 & 15037.8 & 15.339 & 15037.8 & 15.339 & 15037.8 & 15.339 & 15459.9 & 12.963 \\
\hline case24_3zones_acdc & 150228 & $*$ & $*$ & 150156.0 & 0.0479 & 150156.0 & 0.0479 & 150156.0 & 0.0479 & 143715.0 & 4.336 \\
\hline case5_dcgrid, $b_{c}^{c v} \neq 0$ & 55.2914 & 55.2895 & 0.003 & 55.2893 & 0.004 & 55.2892 & 0.004 & 55.2895 & 0.003 & 52.283 & 5.44 \\
\hline case5_dcgrid, $b_{c}^{c v}=0$ & 55.2145 & 55.2139 & 0.001 & 55.2142 & 0 & 55.2142 & 0 & 55.2145 & 0 & 52.206 & 5.449 \\
\hline case5_b2bdc & 193.019 & 193.006 & 0.007 & 182.833 & 5.277 & 182.833 & 5.277 & 182.834 & 5.277 & 172.791 & 10.48 \\
\hline case39_acdc & 41968.9 & $*$ & $*$ & 41961.8 & 0.017 & 41961.8 & 0.017 & 41961.8 & 0.017 & 41114.8 & 2.035 \\
\hline case588_sdet_acdc & 378635 & $*$ & $*$ & 372649 & 1.58083 & 371842 & 1.79403 & 371842 & 1.79393 & 369264 & 2.47503 \\
\hline case3120sp_acdc & 2142635 & * & $*$ & 2131071 & 0.539691 & 2130962 & 0.544804 & 2131001 & 0.54299 & 2080635 & 2.89365 \\
\hline
\end{tabular}

TABLE V

AC/DC OPF COMPUTATION TIME. (NUMERICAL ISSUE INDICATED *)

\begin{tabular}{lrrrrrr}
\hline case & $\begin{array}{r}\text { BIM NLP } \\
\text { time (s) }\end{array}$ & $\begin{array}{r}\text { SDP } \\
\text { time (s) }\end{array}$ & $\begin{array}{r}\text { QC } \\
\text { time (s) }\end{array}$ & $\begin{array}{r}\text { BIM SOC } \\
\text { time (s) }\end{array}$ & $\begin{array}{r}\text { BFM SOC } \\
\text { time (s) }\end{array}$ & $\begin{array}{r}\text { 'DC' } \\
\text { time (s) }\end{array}$ \\
\hline case5_2grids & 0.203682 & 0.093 & 0.198218 & 0.0856372 & 0.0461354 & 0.0130507 \\
case5_acdc & 0.169564 & 0.047 & 0.122575 & 0.121855 & 0.0414212 & 0.0169359 \\
case5_lcc & 0.211576 & 0.031 & 0.143454 & 0.076071 & 0.0491643 & 0.0196013 \\
case5_dc & 0.168613 & 0.031 & 0.0571084 & 0.0532473 & 0.0374073 & 0.025437 \\
case24_3zones_acdc & 1.68523 & $*$ & 1.88709 & 2.13047 & 0.259193 & 0.0706135 \\
case5_dcgrid, bc cv $\neq 0$ & 0.098082 & 0.032 & 0.104582 & 0.168562 & 0.0494967 & 0.0736263 \\
case5_dcgrid, bc cv $=0$ & 0.210359 & 0.031 & 0.17583 & 0.0830827 & 0.0465005 & 0.0303707 \\
case5_b2bdc & 0.14691 & 0.032 & 0.0899259 & 0.0898848 & 0.0340877 & 0.0115135 \\
case39_acdc & 1.66632 & $*$ & 3.55088 & 1.70781 & 0.810524 & 0.0366849 \\
case588_sdet_acdc & 29.0993 & $*$ & 40.568 & 39.7236 & 2.57177 & 0.258169 \\
case3120sp_acdc & 122.725 & $*$ & 154.011 & 205.343 & 18.3339 & 2.60336 \\
\hline
\end{tabular}

rely on the formulation of a single problem reflecting the behavior of all components simultaneously, i.e. it does not iterate between solving ac and dc grids.

Inheriting PowerModels' flexible design, the ac/dc implementation also allows for several possible extensions. Convex formulations can easily be extended with integer variables, e.g. to model converter efficiency depending on rectifier or inverter mode, discrete transformer tap settings or network expansion planning.

Furthermore, by modeling failure of converters and dc lines as well as droop actions of the converters in contingency situations, a security-constrained OPF extension can be developed. The formulations can easily be extended to multiconductor dc grids, in order to generalize to bipolar dc grids with monopolar power injections.

\section{REFERENCES}

[1] J. De Decker and P. Kreutzkamp, "Offshore electricity grid infrastructure in Europe - final report," 3E, Tech. Rep., Oct. 2011.

[2] PROMOTioN. Progress on meshed HVDC offshore transmission networks, European Union Horizon 2020 research and innovation programme, project ID: 691714. https://www.promotion-offshore.net/.

[3] Best Paths. Beyond state-of-the-art technologies for power AC corridors and multi-terminal HVDC systems, European Union Horizon 2020 research and innovation programme, project ID: 612748 . http://www.bestpaths-project.eu/.
[4] InnoDC. Innovative tools for offshore wind and DC grids, European Union Horizon 2020 research and innovation programme, Project ID: 765585. http://innodc.org/.

[5] M. M. El-marsafawy and R. M. Mathur, "A new, fast technique for load-flow solution of integrated multi-terminal DC/AC systems," IEEE Trans. Power Appar. Syst., vol. 75, no. 1, pp. 246-255, 1980.

[6] J. Beerten, "MatACDC 1.0 User's Manual," pp. 1-36, 2012. [Online]. Available: https://www.esat.kuleuven.be/electa/teaching/matacdc/MatACDCManual

[7] C. Lu, S. S. Chen, and C. M. Ong, "The incorporation of HVDC equations in optimal power flow methods using sequentia quadratic programming techniques," IEEE Trans. Power Syst., vol. 3, no. 3, pp. 1005-1011, 1988.

[8] R. Wiget and G. Andersson, "Optimal power flow for combined AC and multi-terminal HVDC grids based on VSC converters," in IEEE Power Energy Soc. General Meeting, San Diego, CA, USA, 2012, pp. 1-8.

[9] M. Aragüés-Peñalba, A. Egea-Àlvarez, O. Gomis-Bellmunt, and A. Sumper, "Optimum voltage control for loss minimization in HVDC multi-terminal transmission systems for large offshore wind farms," Electric Power Syst. Research, vol. 89, pp. 54-63, aug 2012.

[10] J. Rimez and R. Belmans, "A combined ac/dc optimal power flow algorithm for meshed ac and dc networks linked by vsc converters," Int. Trans. Elec. Energy Syst., vol. 25, no. 10, pp. 2024-2035, 2015.

[11] R. D. Zimmerman, C. E. Murillo-Sánchez, and R. J. Thomas, "MATPOWER: steady-state operations, systems research and education," IEEE Trans. Power Syst., vol. 26, no. 1, pp. 12-19, 2011.

[12] W. Feng, L. A. Tuan, L. B. Tjernberg, A. Mannikoff, and A. Bergman, "A new approach for benefit evaluation of multiterminal VSCHVDC using a proposed mixed AC/DC optimal power flow," IEEE Trans. Power Del., vol. 29, no. 1, pp. 432-443, 2014.

[13] E. Iggland, R. Wiget, S. Chatzivasileiadis, and G. Anderson, "Multi- 
area DC-OPF for HVAC and HVDC grids," IEEE Trans. Power Syst. vol. 30, no. 5, pp. 2450-2459, 2015.

[14] R. Wiget and G. Andersson, "Dc optimal power flow including hvdc grids," in IEEE Electrical Power Energy Conf., Aug 2013, pp. 1-6.

[15] F. Geth, R. D'hulst, and D. Van Hertem, "Convex power flow models for scalable electricity market modelling," in CIRED, Glasgow, Scotland, 2017, pp. 1-5.

[16] S. H. Low, "Convex relaxation of optimal power flow - part I: formulations and equivalence," IEEE Trans. Control Netw. Syst., vol. 1, no. 1, pp. 15-27, mar 2014.

[17] L. Gan and S. H. Low, "Optimal power flow in direct current networks," IEEE Trans. Power Syst., vol. 29, no. 6, pp. 2892-2904, 2014.

[18] Z. Yang, H. Zhong, A. Bose, Q. Xia, and C. Kang, "Optimal power flow in AC-DC grids with discrete control devices," IEEE Trans. Power Syst., vol. 33, no. 2, pp. 1461-1472, 2018.

[19] S. Bahrami and V. W. Wong, "Security-constrained unit commitment for ac-dc grids with generation and load uncertainty," IEEE Trans. Power Syst., vol. 33, no. 3, pp. 2717 - 2732, 2018.

[20] S. Bahrami, F. Therrien, V. W. S. Wong, and J. Jatskevich, "Semidefinite relaxation of optimal power flow forac-dc grids," IEEE Trans. Power Syst., vol. 32, no. 1, pp. 289 - 304, 2017.

[21] C. Coffrin, R. Bent, K. Sundar, Y. Ng, and M. Lubin, "PowerModels.j1: an open-source framework for exploring power flow formulations," in Power Syst. Comp. Conf., vol. 20, Dublin, Ireland, 2018, p. 8.

[22] I. Dunning, J. Huchette, and M. Lubin, "JuMP: a modeling language for mathematical optimization," SIAM Review, vol. 59, no. 2, pp. 295-320, 2017.

[23] H. Ergun, F. Geth, and D. Van Hertem, "PowerModelsACDC repository," 2018. [Online]. Available: https://github.com/hakanergun/PowerModelsACDC.j1

[24] J. Beerten, S. Cole, and R. Belmans, "Generalized steady-state VSC MTDC model for sequential AC / DC power flow algorithms," IEEE Trans. Power Syst., vol. 27, no. 2, pp. 821-829, 2012.

[25] Z. Zhao and W. Bin, "Influence of transformer tap-changer control mode upon HVDC valve power loss," in IEEE Power Energy Soc. General Meeting, National Harbor, MD, USA, 2014, pp. 1-4.

[26] M. P. Bahrman and B. K. Johnson, "The ABCs of HVDC transmission technologies," IEEE Power Energy Mag., vol. 5, no. 2, pp. 32-44, 2007.

[27] F. Wang and C. Wang, "Parameters' calculation for converter transformer in hvdc system," in China Int. Conf. Electricity Distribution (CICED), Sept 2014, pp. 26-29.

[28] Markus Pettersson. (2016, September) ABB HVDC Converter Transformers http://www02.abb.com/global/seitp/seitp202.nsf/0/be0fae657152c39dc 125803d0012dd70/\$file/HVDC+converter+transformers.pdf.

[29] W. Wang and M. Barnes, "Power flow algorithms for multi-terminal vsc-hvdc with droop control," IEEE Trans. Power Syst., vol. 29, no. 4, pp. 1721-1730, July 2014.

[30] J. Rimez, "Optimal operation of hybrid AC / DC meshed grids," PhD Dissertation, University of Leuven (KU Leuven), 2014.

[31] G. Daelemans, K. Srivastava, M. Reza, S. Cole, and R. Belmans, "Minimization of steady-state losses in meshed networks using vsc hvdc," in IEEE Power Energy Soc. General Meeting, July 2009, pp. $1-5$.

[32] P. Kundur, N. J. Balu, and M. G. Lauby, Power system stability and control. McGraw-hill New York, 1994, vol. 7.

[33] X. Bai, H. Wei, K. Fujisawa, and Y. Wang, "Semidefinite programming for optimal power flow problems," Int. J. Elec. Power \& Energy Syst., vol. 30, no. 6-7, pp. 383-392, jul 2008.

[34] C. Coffrin, H. Hijazi, and P. Van Hentenryck, "The QC relaxation: a theoretical and computational study on optimal power flow," IEEE Trans. Power Syst., vol. 31, no. 4, pp. 3008-3018, 2016.

[38] E. D. Andersen and K. D. Andersen, "The mosek interior point optimizer for linear programming: an implementation of the homogeneous algorithm," in High performance opt. Springer, 2000, pp. 197-232.
[35] A. Wächter and L. T. Biegler, "On the implementation of primaldual interior point filter line search algorithm for large-scale nonlinear programming," Math. Prog., vol. 106, no. 1, pp. 25-57, 2006.

[36] S. H. Low, "Convex relaxation of optimal power flow - part II: exactness," IEEE Trans. Control Netw. Syst., vol. 1, no. 2, pp. 177-189, 2014.

[37] C. Coffrin, H. Hijazi, and P. Van Hentenryck, "Strengthening the SDP relaxation of ac power flows with convex envelopes, bound tightening, and valid inequalities," IEEE Trans. Power Syst., vol. 32, no. 5, pp. 3549-3558, 2017.

[39] R. Diao, "A 500 bus model created by the sustainable data evolution technology (sdet) tool," IEEE PES Power Grid Library - Optimal Power Flow v18.08, Aug. 2018.

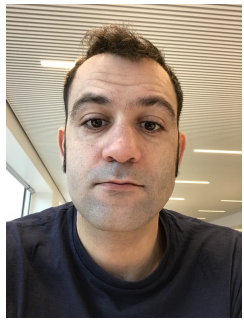

Hakan Ergun (S'10, M'15) received the master degree in electrical engineering (Dipl.-Ing.) at the TU Graz, Austria, in 2009. In 2015, he obtained the $\mathrm{Ph} . \mathrm{D}$. degree from KU Leuven, Belgium. He is a currently a research expert at KU Leuven/EnergyVille working on transmission expansion planning for large scale renewable integration, power system security and optimization models in power systems. $\mathrm{He}$ is an active member of IEEE and the chair of the IEEE PES/PELS/IAS Benelux Chapter.

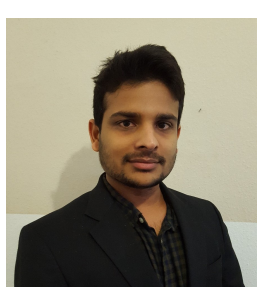

Jaykumar Dave was born in Ahmedabad, India, in 1987. He received his B.Eng. degree in electrical engineering from S.P. University in 2009 and M.Sc. degree in electrical power engineering from RWTH Aachen University in 2016. He is currently a Ph.D. student at KU Leuven, Belgium. His areas of interests are long term transmission system planning and HVDC grid protection.

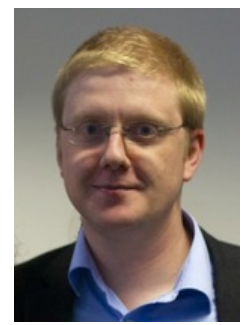

Dirk Van Hertem Dirk Van Hertem (S?02-SM?09) was born in 1979, in Neerpelt, Belgium. He received the M.Eng. from the Katholieke Hogeschool der Kempen, Geel, Belgium in 2001 and the M.Sc. and $\mathrm{Ph} . \mathrm{D}$. degrees in electrical engineering from the University of Leuven, Leuven, Belgium in 2003 and 2009, respectively. His special fields of interest are power system operation and control in systems with flexible ac transmission systems and HVDC and building the transmission system of the future, including offshore grids and the supergrid concept. Since 2011, he has been with the University of Leuven, Leuven, where he is an Associate Professor in the Electa group. He is an active member of the IEEE PES, IAS and Cigré.

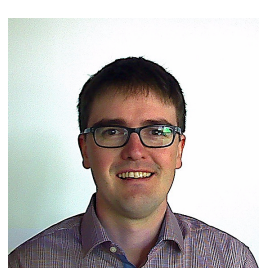

Frederik Geth (S'10, M'17) received the M.Sc. degree in Electrical Engineering from the KU Leuven, Leuven, Belgium, in 2009 and the Ph.D. degree in 2014. Since 2018 he is a research scientist with CSIRO Energy in Newcastle Australia. His research interests include modeling of the integration of electric vehicles and storage in distribution grids specifically, and optimal power flow models in general. 